\title{
EFECTOS GENÉTICOS EN CRUZAS DIRECTAS Y RECÍPROCAS FORMADAS A PARTIR DE LÍNEAS DE DOS GRUPOS GERMOPLÁSMICOS DE MAÍZ
}

\author{
GENETIC EFFECTS OF DIRECT AND RECIPROCAL CROSSES OBTAINED FROM TWO \\ GERMPLASM GROUP LINES IN MAIZE
}

\author{
Daniel Sámano Garduño ${ }^{1}$, Froylán Rincón Sánchez ${ }^{2 *}$, Norma A. Ruiz Torres ${ }^{3}$, José Espinoza Velázquez ${ }^{4}$ y \\ Humberto De León Castillo ${ }^{1}$
}

${ }^{1}$ Instituto Mexicano del Maíz, Universidad Autónoma Agraria Antonio Narro (UAAAN). 25315, Buenavista, Saltillo, Coahuila, México. ${ }^{2}$ Departamento de Fitomejoramiento, UAAAN. ${ }^{3}$ Centro de Capacitación y Desarrollo de Tecnología de Semillas, UAAAN.

*Autor para correspondencia (frincon@uaaan.mx)

\section{RESUMEN}

En este trabajo se analizó la expresión fenotípica y genotípica de 90 cruzas simples directas (A x B) de maíz (Zea mays $\mathbf{L}$.) formadas por el apareamiento de 10 líneas del Grupo Enano (A) con nueve líneas normales (B) QPM (Quality Protein Maize), más las 90 cruzas recíprocas $(B \times \mathbf{A})$. Las cruzas se evaluaron en tres ambientes contrastantes en 2004. Se utilizó el promedio de altura de planta $(\mathrm{cm})$ y rendimiento de mazorca $\left(t \mathbf{h a}^{-1}\right)$ a través de localidades para analizar la expresión de las cruzas, y estimar la aptitud combinatoria de líneas y cruzas en las combinaciones A $x$ B y B $x$ A. Se encontraron diferencias $(P \leq 0.01)$ entre cruzas, así como en los componentes de los grupos A x B y B x A, en las líneas, probadores y la interacción líneas $\mathrm{x}$ probador dentro de cada grupo, para las dos variables en estudio, así como en el contraste entre los grupos A x B vs. B x A. Esto indica la variabilidad de las líneas en cada grupo, la importancia de su interacción y la diferencia ente los efectos directos y recíprocos en cruzas. La expresión de las cruzas entre líneas de los dos grupos germoplásmicos estuvo determinada por efectos aditivos de las líneas QPM, principalmente con 70.2 y $61.5 \%$ de la variación en altura de planta y rendimiento de mazorca, respectivamente. Al combinar líneas entre estos dos grupos germoplásmicos no emparentados genéticamente, cambió el orden de participación de los progenitores y determinó diferencias en la expresión fenotípica, y en la estimación de los efectos genéticos de las líneas y sus cruzas. El orden de cruzamiento modificó los valores estimados de aptitud combinatoria general en el Grupo Enano y los efectos no-aditivos de los dos grupos de líneas, ya que en la aptitud combinatoria específica se obtuvieron correlaciones del orden de $r_{s}=0.184$ y $r_{s}=0.252 *$ para altura de planta y rendimiento de mazorca.

Palabras clave: Zea mays, aptitud combinatoria, efectos recíprocos.

\section{SUMMARY}

The phenotypic and genotypic expression of 90 single maize (Zea mays L.) crosses (A $\times$ B) obtained by crossing 10 lines from the dwarf group (A) with nine QPM normal lines (B), plus the 90 reciprocal crosses $(B \times A)$, were analyzed in this work. Crosses were evaluated in three contrasting environments in 2004. The average performance across locations was used to analyze the crosses expression, and to estimate the combining ability of lines and crosses on the A $\times B$ and B $\times$ A combinations for plant height $(\mathrm{cm})$ and ear yield $\left(\mathrm{t} \mathrm{ha}^{-1}\right)$. Significant differences $(P \leq 0.01)$ among crosses were found, as well as in their group components $A \times B$ and $B \times A$ in lines, testers and the line by tester interaction within each group for the two traits analyzed, including the groups A $\times$ B vs. B x A contrast. Results indicated variability within each group, the meaning of their interaction, and the difference between the direct and reciprocal effects of crosses. The crosses expression between lines from the two germplasm groups were determined by additive effects, mainly in the QPM lines with 70.2 and $61.5 \%$ of the explained variation for plant height and ear yield, respectively. In the combination of lines among genetically unrelated germplasm groups, a modification in the participation order of parental lines determines the differences in the phenotypic expression, and in the estimation of lines and crosses genetic effects. The values of the general combining ability estimates were modified by the crossing order in the dwarf group, and the non-additive effects of the lines from the two groups, as correlations of $r_{s}=\mathbf{0 . 1 8 4}$ and $r_{s}=0.252 *$ were obtained for plant height and ear yield.

Index words: Zea mays, combining ability, reciprocal effects.

\section{INTRODUCCIÓN}

Los programas de mejoramiento genético dedicados a la formación de híbridos y variedades comerciales de maíz (Zea mays L.), requieren generar nuevas líneas con alto potencial de rendimiento, buen comportamiento agronómico y excelente aptitud combinatoria; las líneas que reúnen tales características han demostrado resultados satisfactorios en combinaciones híbridas (Fan et al., 2003). Asimismo, se pueden utilizar líneas de diferente origen genético para maximizar la respuesta heterótica y formar patrones heteróticos sobresalientes que simplifiquen la 
formación de híbridos y variedades sintéticas de maíz (Bernardo, 2001; Hoxha et al., 2004; Li et al., 2004). La aptitud combinatoria de líneas endogámicas de maíz puede calcularse a través de cruzas de prueba con probadores divergentes, cuyo comportamiento permita estimar la distancia genética entre ellas, así como clasificarlas en grupos heteróticos (Soengas et al., 2003).

La evaluación y selección de líneas es la etapa de mayor relevancia en el proceso de mejoramiento, ya que además de identificar las mejores líneas permite encontrar la mejor combinación híbrida para explotar la heterosis. También es importante que las líneas e híbridos se evalúen en diferentes localidades para seleccionar los genotipos con mayor estabilidad (Coutiño y Vidal, 2003).

Tradicionalmente, en los estudios de cruzas de maíz se hace énfasis sólo en la aptitud combinatoria general (ACG) y específica (ACE), y se ignoran los efectos recíprocos y maternos. Al respecto, Corey et al. (1976), Kang et al. (1999), Dhliwayo et al. (2005) y Haro et al. (2007) encontraron diferencias en la expresión fenotípica de cruzas en cultivos como maíz, girasol (Helianthus annuus) y Arabidopsis thaliana, en características como acame de tallo, resistencia a plagas, rendimiento de grano y en la calidad física y química de la semilla, debido a que los patrones de herencia están controlados por efectos maternos y paternos. Cuando se analizan los efectos recíprocos, se puede tomar en cuenta líneas de un mismo complejo genético, pero hay poca o nula información sobre cruzas $\mathrm{F}_{1}$ formadas con líneas que difieren en al menos un carácter.

El objetivo de esta investigación fue estudiar el efecto que genera en altura de planta y rendimiento de mazorca, el cambio de dirección del cruzamiento entre líneas derivadas de dos grupos germoplásmicos no emparentados genéticamente, en la expresión fenotípica y genotípica de sus cruzas simples.

\section{MATERIALES Y MÉTODOS}

El trabajo de investigación involucró 10 líneas endogámicas (identificadas con los números 1 al 10) derivadas del grupo de maíz enano (Grupo A) del Programa Bajío del Instituto Mexicano del Maíz "Dr. Mario E. Castro Gil” (IMM) de la Universidad Autónoma Agraria Antonio Narro (UAAAN), y nueve líneas endogámicas (identificadas con los números 11 al 19) QPM (Quality Protein Maize) procedentes del programa del subtrópico (Grupo B) del Centro Internacional de Mejoramiento de Maíz y Trigo (CIMMYT).
Las líneas del IMM son se de grano blanco semicristalino, cuyas plantas tienen entrenudos cortos abajo de la mazorca, hojas erectas y espiga compacta; características que les permiten soportar altas densidades de población. Las líneas tienen ciclo de madurez precoz a intermedio y se adaptan al subtrópico de México (ambientes tipo "Bajío"). Las líneas QPM del CIMMYT se adaptan al subtrópico, producen grano blanco cristalino, semicristalino, dentado y semidentado, son de ciclo de madurez intermedio a tardío y fueron seleccionadas por su contenido de proteína (CIMMYT, 1998).

En el ciclo agrícola otoño-invierno (O-I) 2003-2004 de Tepalcingo, Mor. se hicieron los cruzamientos de prueba entre los dos grupos de líneas, en donde las líneas del Grupo A se cruzaron en forma directa y recíproca con las del Grupo B (probador), y se generaron 180 cruzas, 90 A $\mathrm{x}$ B y $90 \mathrm{~B}$ x A.

Las 180 cruzas simples se evaluaron en el ciclo agrícola primavera-verano (PV) de 2004 en tres localidades: dos en El Bajío (Juventino Rosas, Gto. y La Piedad, Mich., con 1697 y 1695 m de altitud, respectivamente) y la otra en el norte del país (El Prado, Galeana, N. L., con $1890 \mathrm{~m}$ de altitud). La unidad experimental consistió en un surco de $4 \mathrm{~m}$ de largo por $0.75 \mathrm{~m}$ de ancho en El Bajío y de $0.92 \mathrm{~m}$ en El Prado, con 21 plantas por surco y dos repeticiones por localidad. Se utilizó el diseño experimental de bloques incompletos en arreglo $\alpha$-Látice. La siembra se llevó a cabo en forma manual; se depositaron dos semillas por golpe para después aclarear a 70000 y 57000 plantas ha $^{-1}$ en El Bajío y El Prado, respectivamente. Las variables estudiadas fueron altura de planta (cm) y rendimiento de mazorca $\left(\mathrm{t} \mathrm{ha} \mathrm{h}^{-1}\right)$ a $15.5 \%$ de humedad.

Los datos obtenidos en cada tipo de cruza (A x B y B $\mathrm{x}$ A) se analizaron con el programa estadístico SAS versión 9.1 (SAS Institute, 2004) al combinar las tres localidades. El comportamiento promedio de las cruzas directas y recíprocas ( $\mathrm{A} \times \mathrm{B}$ y $\mathrm{B} \times \mathrm{A})$, a través de los ambientes de evaluación, se analizó mediante la estimación de las diferencias en las combinaciones lineales de los valores observados correspondientes a cada grupo de cruzas $(\mathrm{KY})$, con la esperanza $E(K Y)=K X \beta$; donde $\mathrm{KY}$ corresponde a los vectores de coeficientes asociados con los valores observados; $\mathrm{X}$ y $\beta$, la matriz de diseño y el vector de parámetros del modelo, respectivamente (SAS Institute, 2004). Cada combinación lineal tiene asociado un error estándar (EE), el cual se usó para una prueba de t, como sigue: $t=\left(\mu_{1}-\mu_{2}\right) / E E\left(\mu_{1}-\mu_{2}\right)$.

Los cruzamientos entre líneas de los dos grupos (Enano y QPM) fueron analizados con el modelo "línea por 
probador" descrito por Singh y Chaudhary (1985), donde se consideró por un lado al grupo Enano como hembra y al grupo QPM como probador (AB) y, por el otro al grupo QPM como hembra y al grupo Enano como macho (probador). La aptitud combinatoria general (ACG) de líneas y probadores, así como la aptitud combinatoria específica (ACE) para cada tipo de cruzas se estimaron de la siguiente manera: $g_{i}=\mu_{i .}-\mu ; g_{j}=\mu_{. j}-\mu$; $s_{i j}=\mu_{i j}-\mu-g_{i}-g_{j}$; donde $g_{i}$ y $g_{j}$ son los efectos de ACG para los progenitores $i, j$, respectivamente; $s_{i j}$ el efecto de ACE de la cruza $i \mathrm{x} j ; \mu_{i .}, \mu_{. \mathrm{j}}$ y $\mu$ son la media de las cruzas donde interviene el progenitor $i, j$ y la media general, respectivamente. En las pruebas de hipótesis de los efectos de aptitud combinatoria en las combinaciones A x B y B x A, se utilizó el cuadrado medio de la interacción con localidades para calcular el error estándar que refleje la respuesta de las líneas y sus combinaciones a través de ambientes.

Debido a que las líneas y cruzas tuvieron un estimado de ACG y ACE diferente al ser considerados como machos o como hembras, se calculó el coeficiente de correlación de Spearman (SAS Institute, 2006) para determinar el grado de asociación de los estimados al invertir el sen- tido de la cruza. El coeficiente se calculó con la ecuación siguiente: $r_{s}=1-6 \Sigma \frac{d^{2}}{n\left(n^{2}-1\right)}$, en donde $d$ es la diferencia entre los rangos obtenidos en los estimados de ACG y ACE en las cruzas A x B y B x A, respectivamente, y $\mathrm{n}$ el número de observaciones.

\section{RESULTADOS Y DISCUSIÓN}

En el análisis de varianza (Cuadro 1) hubo diferencias $(\mathrm{P} \leq 0.01)$ para localidades en las dos variables evaluadas, lo que indica que entre los tres ambientes de prueba existen condiciones que hace que se diferencien. Se encontró diferencia $(\mathrm{P} \leq 0.01)$ entre las cruzas evaluadas (Cuadro 1) en altura de planta (AP) y rendimiento (REND), lo cual podría deberse a la variabilidad genética que existe entre las líneas de ambos grupos, así como al diferente grado de combinación entre ellas. La descomposición de la suma de cuadrados de las cruzas en los grupos A x B y B x A, líneas, probadores e interacción dentro de cada grupo, mostraron diferencias $(\mathrm{P} \leq 0.01)$ en las dos variables bajo estudio, que incluyen el contraste entre los grupos A x B vs. B x A.

Cuadro 1. Análisis de varianza combinado entre dos grupos germoplásmicos para las variables altura de planta (AP) y rendimiento de mazorca a 15 \% de humedad (REND), evaluados en el ciclo agrícola primavera-verano de 2004.

\begin{tabular}{|c|c|c|c|c|c|}
\hline \multirow[b]{2}{*}{ Fuentes de variación } & \multirow[b]{2}{*}{$\mathrm{gl}$} & \multicolumn{2}{|c|}{ Altura de planta $(\mathrm{cm})$} & \multicolumn{2}{|c|}{ REND (t ha $\left.{ }^{-1}\right)$} \\
\hline & & $\mathrm{SC}$ & $\mathrm{CM}$ & $\mathrm{SC}$ & $\mathrm{CM}$ \\
\hline Localidades (Loc) & 2 & 534346.98 & $267173.49 * *$ & 1292.81 & $646.41 * *$ \\
\hline Bloques/Loc & 3 & 2003.65 & 667.88 & 28.14 & $9.38 * *$ \\
\hline Cruzas & 179 & 455842.50 & $2546.61^{* *}$ & 2613.32 & $14.60 * *$ \\
\hline $\mathrm{A} \times \mathrm{B}$ & 89 & 195376.64 & $2195.24 * *$ & 937.62 & $10.54 * *$ \\
\hline Líneas A & 9 & 37618.16 & $4179.80^{* *}$ & 69.66 & $7.74 * *$ \\
\hline Probador B & 8 & 116603.24 & $14575.40 * *$ & 470.43 & $58.80 * *$ \\
\hline Línea x Probador & 72 & 41155.24 & $571.60 * *$ & 397.53 & $5.52 * *$ \\
\hline $\mathrm{B} \times \mathrm{A}$ & 89 & 231728.79 & $2603.69 * *$ & 1634.63 & $18.37 * *$ \\
\hline Líneas B & 8 & 187248.14 & $23406.02 * *$ & 1191.54 & $148.94 * *$ \\
\hline Probador A & 9 & 14531.86 & $1614.65^{* *}$ & 103.14 & $11.46 * *$ \\
\hline Línea x Probador & 72 & 29948.79 & $415.96 * *$ & 339.96 & $4.72 * *$ \\
\hline $\mathrm{A} \times \mathrm{B} v s . \mathrm{B} \times \mathrm{A}$ & 1 & 28737.08 & $28737.08 * *$ & 41.07 & $41.07 * *$ \\
\hline Cruzas x Loc & 358 & 257731.02 & $719.92 * *$ & 1839.57 & $5.14 * *$ \\
\hline A x B x Loc & 178 & 184057.42 & $1034.03 * *$ & 1267.93 & $7.12 * *$ \\
\hline Líneas A x Loc & 18 & 48052.34 & $2669.57 * *$ & 331.97 & $18.44 * *$ \\
\hline Probador B x Loc & 16 & 65313.42 & $4082.09 * *$ & 275.91 & $17.24 * *$ \\
\hline Línea x Probador x Loc & 144 & 70691.66 & $490.91 * *$ & 660.05 & $4.58 * *$ \\
\hline B x A x Loc & 178 & 73387.93 & $412.29 * *$ & 540.03 & $3.03 *$ \\
\hline Líneas B x Loc & 16 & 20860.33 & $1303.77 * *$ & 153.93 & $9.62 * *$ \\
\hline Probador A x Loc & 18 & 10146.52 & $563.70 * *$ & 52.87 & 2.94 \\
\hline Línea x Probador x Loc & 144 & 42381.08 & 294.31 & 333.23 & 2.31 \\
\hline Error & 537 & 141054.85 & 262.67 & 1281.64 & 2.39 \\
\hline CV (\%) & . & & 8.53 & 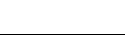 & 12.69 \\
\hline
\end{tabular}


El cambio originado en las cruzas al invertir de orden de participación de los progenitores, se expresó como 42.9 y $50.8 \%$ en la proporción relativa de la suma de cuadrados para las cruzas A x B y B x A en altura de planta, respectivamente. Las proporciones para rendimiento de mazorca, fueron de 35.9 y $62.5 \%$ para las cruzas A x B y B x A, respectivamente. Las diferencias en altura de planta y rendimiento de mazorca entre las cruzas A x B y B x A fueron significativas $(\mathrm{P} \leq 0.01)$. En la descomposición de la suma de cuadrados de altura de planta, las líneas del Grupo Enano contribuyeron con 19.3 y $6.3 \%$ en las cruzas A x B y B x A, respectivamente, en tanto que las líneas del Grupo QPM contribuyeron con 59.7 y $80.8 \%$ en las mismas combinaciones. En rendimiento de mazorca, las líneas del grupo Enano contribuyeron con 7.4 y $6.3 \%$ de la variación en las cruzas A x B y B x A, respectivamente, en tanto que las líneas QPM con 50.2 y $72.9 \%$ de la variación en las cruzas A x B y $\mathrm{B} \times \mathrm{A}$, respectivamente; este comportamiento es similar al encontrado en altura de planta. Por tanto, las líneas enanas que portan el gen braquítico $\left(b r_{2} b r_{2}\right)$ en condición homocigótica recesiva, al aparearse con líneas QPM de porte normal, en promedio contribuyen con 12.8 y $6.9 \%$ de la variación expresada en las combinaciones A x B y B $\mathrm{x}$ A para altura de planta y rendimiento de mazorca, respectivamente (Cuadro 1). Las líneas QPM contribuyen con 70.2 y $61.5 \%$ de la variación en las cruzas A x B y $\mathrm{B}$ x A en altura de planta y rendimiento de mazorca, respectivamente.

Estos resultados establecen que además de la variabilidad genética que presenta cada uno de los grupos, la diversidad genética existente entre ellos influye en el cambio de comportamiento de las cruzas al considerar uno $\mathrm{u}$ otro grupo como hembra o como macho, lo que coincide con lo informado por Pollak et al. (1991) quienes encontraron efectos recíprocos importantes en altura de planta y rendimiento de grano, al comparar las combinaciones heteróticas entre poblaciones de maíz del Caribe y del trópico.

En las dos variables estudiadas, las líneas del Grupo QPM consideradas indistintamente como hembras o como machos, mostraron mayor variación (Cuadro 1) en comparación con las líneas del Grupo Enano. En un estudio previo, los Grupos Enano y QPM mostraron ser una combinación germoplásmica con alto potencial de rendimiento (De León et al., 2005). En el presente trabajo, la expresión tanto en altura de planta y en rendimiento de mazorca se debió a efectos aditivos, principalmente del grupo QPM; sin embargo, los efectos no aditivos representaron 21.1 y $42.4 \%$ de la variación para altura de planta y rendimiento de mazorca, respectivamente, en la combinación de la cruzas A x B, lo cual representa aproximadamente el doble de la variación explicada en comparación con las cruzas B x A (12.9 y 20.8 \%, respectivamente).

La interacción de las cruzas con el ambiente fue mayor en las cruzas A x B (250 y $234 \%$ para altura de planta y rendimiento de mazorca, con diferencias $(\mathrm{P} \leq 0.01)$ en altura de planta y rendimiento de mazorca, mientras que para las cruzas $\mathrm{B} \times \mathrm{A}$, hubo diferencias en altura de planta $(\mathrm{P} \leq 0.01)$ y el rendimiento de mazorca $(\mathrm{P} \leq 0.05)$, lo cual indica que el ambiente influyó en la expresión del rendimiento, principalmente en las cruzas A x B.

El análisis comparativo de la respuesta de las cruzas al considerar las líneas como hembra o como macho para las dos variables, se presentan como una estimación de la diferencia entre los dos tipos de cruzas (Cuadro 2). La significancia estadística basada en la prueba de $t$, indica que en altura de planta 36 cruzas (40.0\%) mostraron diferencias entre A x B y B x A, mientras que en el rendimiento de mazorca sólo 20 cruzas (22.2\%) mostraron diferencias. Es evidente que la mayor diferencia se encontró en altura de planta por ser dos grupos contrastantes (Grupos Enano y QPM normal). Aunque esta diferencia no fue tan marcada en el rendimiento de mazorca, pero sí fue de consideración por su proporción en las comparaciones (22.2\%). Del total de las cruzas con significancia estadística (Cuadro 2), en sólo ocho cruzas $(22.2 \%)$ en altura de planta y cinco $(25.0 \%)$ en rendimiento de mazorca cuando se utilizaron las líneas QPM como hembra, se obtuvieron resultados superiores. Por tanto, se concluye que la combinación más apropiada entre estos dos grupos germoplásmicos es usar líneas del Grupo Enano como hembras (A x B), ya que más de $75 \%$ de las cruzas sobresalientes obtuvieron valores superiores en altura de planta $\mathrm{y}$ mazorca (Cuadro 2).

Estos resultados coinciden con los obtenidos por Kang et al. (1995) y Dhliwayo et al. (2005), quienes encontraron cambios en la aptitud combinatoria general de líneas debido a efectos maternos y no maternos. La importancia de estos resultados es que a pesar de que las líneas del Grupo QPM reflejan una amplia variabilidad genética, mantienen un comportamiento similar al cambiar su participación en las cruzas. Por otro lado, las líneas del Grupo Enano fueron más sensibles a cambiar su expresión genética al ser consideradas como hembras o como machos. Lo anterior se explica por la magnitud de los valores de la ACG y el cambio de signo en algunas líneas en altura de planta (Cuadro 3). En el rendimiento de mazorca, además de la diferencia en la magnitud de los valores entre las cruzas A x B y B x A, se encontró una relación independiente al obtener un coeficiente de correlación de $r_{s}=-0.067$. 
Cuadro 2. Medias de altura de planta y rendimiento de mazorca de cruzas simples directas y recíprocas entre líneas del Grupo Enano (A) y QPM (B), evaluadas en el ciclo agrícola primavera-verano de 2004.

\begin{tabular}{|c|c|c|c|c|c|c|c|c|c|c|c|c|c|c|c|}
\hline \multicolumn{7}{|c|}{ Altura de planta $(\mathrm{cm})$} & \multirow[b]{2}{*}{ Dif } & \multirow[b]{2}{*}{ Cruza } & \multicolumn{7}{|c|}{ Rendimiento de mazorca $\left(\mathrm{t} \mathrm{h}^{-1}\right)$} \\
\hline Cruza & $\mathrm{A} \times \mathrm{B}$ & $\mathrm{B} \times \mathrm{A}$ & Dif & Cruza & $\mathrm{A} \times \mathrm{B}$ & $\mathrm{B} \times \mathrm{A}$ & & & $\mathrm{A} \times \mathrm{B}$ & $\mathrm{B} \times \mathrm{A}$ & Dif & Cruza & $A \times B$ & $\mathrm{~B} \times \mathrm{A}$ & Dif \\
\hline $1 \times 11$ & 189.3 & 177.3 & 12.0 & $6 \times 11$ & 200.7 & 189.5 & 11.2 & 1x11 & 12.09 & 11.17 & 0.92 & $6 \times 11$ & 13.06 & 13.30 & -0.25 \\
\hline $1 \times 12$ & 144.0 & 129.2 & 14.8 & $6 \times 12$ & 174.5 & 135.8 & $38.7^{* *}$ & $1 \times 12$ & 9.11 & 7.91 & 1.20 & $x 12$ & 10.77 & 7.85 & $2.92 * *$ \\
\hline $1 \times 13$ & 209.7 & 197.5 & 12.2 & $6 \times 13$ & 219.3 & 197.7 & $21.7 *$ & $1 \times 13$ & 12.76 & 11.75 & 1.00 & & 12.80 & 12.50 & 0.29 \\
\hline $1 \times 14$ & 203.3 & 191.7 & 11.7 & $6 \times 14$ & 213.3 & 182.5 & $30.8^{* *}$ & $1 \times 14$ & 12.86 & 11.79 & 1.06 & $\mathrm{x} 14$ & 14.19 & 14.10 & 0.10 \\
\hline $1 \times 15$ & 197.7 & 193.7 & 4.0 & $6 \times 15$ & 191.8 & 180.0 & 11.8 & $1 \times 15$ & 14.61 & 13.58 & 1.03 & & 11.46 & 13.13 & -1.67 \\
\hline $1 \times 16$ & 194.0 & 184.7 & 9.3 & $6 \times 16$ & 202.0 & 141.8 & $60.2 * *$ & $1 \times 16$ & 12.96 & 11.88 & 1.08 & $x 16$ & 12.72 & 10.25 & $2.47 * *$ \\
\hline $1 \times 17$ & 194.3 & 186.7 & 7.7 & $6 \times 17$ & 198.7 & 189.3 & 9.3 & $1 \times 17$ & 12.31 & 12.42 & -0.10 & $6 \times 17$ & 12.86 & 12.10 & 0.76 \\
\hline $1 \times 18$ & 190.3 & 210.2 & $-19.8^{*}$ & $6 \times 18$ & 216.3 & 209.3 & 7.0 & $1 \times 18$ & 11.43 & 11.36 & 0.07 & $6 \times 18$ & 12.43 & 11.55 & 0.87 \\
\hline $1 \times 19$ & 194.0 & 183.8 & 10.2 & $6 \times 19$ & 211.7 & 186.3 & $25.3 *$ & $1 \times 19$ & 11.87 & 9.54 & $2.33^{* *}$ & $6 \times 19$ & 12.50 & 11.76 & 0.74 \\
\hline $2 \times 11$ & 186.7 & 196.0 & -9.3 & $7 \times 11$ & 201.7 & 191.7 & 10.0 & $2 \times 11$ & 12.43 & 13.70 & -1.28 & $7 \times 11$ & 12.84 & 12.81 & 0.02 \\
\hline $2 \times 12$ & 148.2 & 143.7 & 4.5 & $7 \times 12$ & 151.0 & 148.3 & 2.7 & $2 \times 12$ & 7.86 & 7.12 & 0.74 & $7 \times 12$ & 10.74 & 9.19 & 1.55 \\
\hline $2 \times 13$ & 192.5 & 208.5 & -16.0 & $7 \times 13$ & 216.3 & 201.7 & 14.7 & $2 \times 13$ & 12.19 & 15.08 & $-2.89 * *$ & $7 \times 13$ & 12.44 & 12.89 & -0.45 \\
\hline $2 \times 14$ & 183.8 & 204.8 & $-21.0^{*}$ & $7 \times 14$ & 202.3 & 198.0 & 4.3 & $2 \times 14$ & 11.70 & 14.51 & $-2.81 * *$ & $7 \times 14$ & 12.78 & 13.53 & -0.75 \\
\hline $2 \times 15$ & 178.5 & 203.8 & $-25.3 * *$ & $7 \times 15$ & 197.3 & 194.5 & 2.8 & $2 \times 15$ & 12.63 & 13.93 & -1.30 & $7 \times 15$ & 14.52 & 14.56 & -0.04 \\
\hline $2 \times 16$ & 166.7 & 190.7 & $-24.0^{*}$ & $7 \times 16$ & 200.8 & 184.3 & 16.5 & $2 \times 16$ & 10.40 & 12.86 & $-2.46 * *$ & $7 \times 16$ & 11.02 & 12.61 & -1.59 \\
\hline $2 \times 17$ & 204.7 & 191.7 & 13.0 & $7 \times 17$ & 199.8 & 185.3 & 14.5 & $2 \times 17$ & 13.07 & 12.56 & 0.50 & $7 \times 17$ & 12.14 & 12.31 & -0.17 \\
\hline $2 \times 18$ & 184.8 & 219.3 & $-34.5^{* *}$ & $7 \times 18$ & 214.8 & 193.0 & $21.8^{*}$ & $2 \times 18$ & 13.30 & 13.82 & -0.51 & $7 \times 18$ & 11.99 & 12.61 & -0.63 \\
\hline $2 \times 19$ & 178.0 & 196.7 & $-18.7^{*}$ & $7 \times 19$ & 200.3 & 190.3 & 10.0 & $2 \times 19$ & 11.85 & 12.40 & -0.54 & $7 \times 19$ & 11.96 & 12.15 & -0.18 \\
\hline $3 \times 11$ & 179.5 & 192.5 & -13.0 & $8 \times 11$ & 204.3 & 177.5 & & $3 \times 11$ & 12.19 & 10.81 & 1.38 & $8 \times 11$ & 12.76 & 13.31 & -0.55 \\
\hline $3 \times 12$ & 131.8 & 124.2 & 7.7 & $8 \times 12$ & 169.2 & 135.8 & & $3 \times 12$ & 9.65 & 8.07 & 1.58 & $8 \times 12$ & 9.08 & 8.28 & 0.80 \\
\hline $3 \times 13$ & 229.8 & 200.3 & 29.5 & & & & & & 12.40 & 12.46 & -0.06 & & 14.06 & 12.65 & 1.41 \\
\hline $3 \times 14$ & 210.0 & 200.0 & 10.0 & & & & & & 2.78 & & & & 12.33 & 12.61 & -0.28 \\
\hline $3 \times 15$ & 2.5 & 190.0 & 12.5 & & & & & & & & & & .08 & & 0.89 \\
\hline $3 \times 16$ & 2 & 18 & 19. & & & & & & & & & & 47 & & 0.10 \\
\hline $3 \times 17$ & .7 & 18 & 17.3 & & & 179.2 & & & 14.38 & & & & 11.86 & & -0.24 \\
\hline $3 \times 18$ & 217.3 & 197.0 & $20.3^{*}$ & & & 206.7 & 0 . & & 13.42 & & -1.7 & 18 & 12.38 & 12.84 & -0.46 \\
\hline $3 \times 19$ & 215.7 & 163.0 & $52.7 * *$ & $8 x$ & 219.2 & 189.7 & 29. & $3 \times 19$ & 11.87 & 11.72 & 0.1 & 19 & 12.84 & 11.91 & 0.93 \\
\hline $4 \times 11$ & 204.5 & 188.8 & 15.7 & $9 \mathrm{x}$ & 198.0 & 167.3 & 30. & $4 \times 11$ & 12.88 & 13.81 & -0.92 & 11 & 12.62 & 12.81 & -0.19 \\
\hline $4 \times 12$ & 179.2 & 142.0 & 37.2 ** & $9 \times 12$ & 156.7 & 125.0 & $31.7 * *$ & $4 \times 12$ & 10.97 & 8.80 & $2.16^{*}$ & $9 \times 12$ & 10.31 & 8.91 & 1.40 \\
\hline $4 \times 13$ & 205.0 & 198.7 & 6.3 & $9 \times 13$ & 224.7 & 197.3 & $27.3^{* *}$ & $4 \times 13$ & 11.55 & 12.60 & -1.05 & $9 \times 13$ & 14.22 & 12.34 & $1.88^{*}$ \\
\hline $4 \times 14$ & 207.3 & 198.3 & 9.0 & $9 \times 14$ & 224.3 & 194.2 & $30.2 *$ & $4 \times 14$ & 14.65 & 14.58 & 0.07 & $9 \times 14$ & 14.48 & 11.87 & $2.62 * *$ \\
\hline $4 \times 15$ & 211.3 & 183.2 & $28.2^{* *}$ & $9 \times 15$ & 211.3 & 206.8 & 4.5 & $4 \times 15$ & 13.37 & 11.37 & $2.00 *$ & $9 \times 15$ & 12.45 & 13.50 & -1.05 \\
\hline $4 \times 16$ & 197.2 & 182.0 & 15.2 & $9 \times 16$ & 199.0 & 180.7 & $18.3^{*}$ & $4 \times 16$ & 12.73 & 12.32 & 0.41 & $9 \times 16$ & 13.91 & 12.06 & $1.85^{*}$ \\
\hline $4 \times 17$ & 196.2 & 183.0 & 13.2 & $9 \times 17$ & 217.7 & 181.7 & $36.0^{* *}$ & $4 \times 17$ & 12.44 & 12.02 & 0.42 & $9 \times 17$ & 14.45 & 11.76 & $2.69^{* *}$ \\
\hline $4 \times 18$ & 203.0 & 178.8 & $24.2 *$ & $9 \times 18$ & 199.5 & 189.3 & 10.2 & $4 \times 18$ & 12.96 & 11.15 & $1.80^{*}$ & $9 \times 18$ & 12.32 & 12.07 & 0.25 \\
\hline $4 \times 19$ & 197.3 & 187.7 & 9.7 & $9 \times 19$ & 207.8 & 186.3 & $21.5^{*}$ & $4 \times 19$ & 11.53 & 11.86 & -0.32 & $9 \times 19$ & 11.99 & 11.60 & 0.40 \\
\hline $5 \times 11$ & 213.7 & 187.0 & $26.7^{* *}$ & $10 \times 11$ & 178.7 & 204.5 & $-25.8^{* *}$ & $5 \times 11$ & 13.08 & 14.41 & -1.33 & $10 \times 11$ & 11.76 & 12.07 & -0.31 \\
\hline $5 \times 12$ & 148.3 & 125.0 & $23.3 *$ & $10 \times 12$ & 156.3 & 14 & 11.3 & $5 \mathrm{x}$ & 10.31 & 6.51 & $3.80 * *$ & $10 \times 12$ & 10.32 & 7.29 & $3.03^{* *}$ \\
\hline $5 \times 13$ & 208.3 & 195.3 & 13.0 & $10 x$ & & & -13 & & 13.69 & 12.28 & 1.40 & & 10.91 & 12.23 & -1.32 \\
\hline $5 \times 14$ & & 183.3 & 12.5 & & & 20 & -12 & & & & 0. & & 11.61 & 11.40 & 0.21 \\
\hline $5 \times 15$ & & & 12.2 & & & & & & & & & & 12.49 & & $-1.94 *$ \\
\hline $5 \times 16$ & & 178.7 & $23.8^{*}$ & & & & & & & & & & 12.40 & & 0.44 \\
\hline $5 \times 17$ & & 179.7 & 4.8 & & & & & & & & & & 13.30 & & $1.98^{*}$ \\
\hline $5 \times 18$ & 210.0 & 201.3 & 8.7 & & & & & & & & & & 12.78 & 1.96 & 0.82 \\
\hline $5 \times 19$ & 179.0 & 184.3 & -5.3 & $10 \times 19$ & 176.0 & 193.7 & -17.7 & $5 \times 19$ & 10.53 & 11.54 & -1.02 & $10 \times 19$ & 11.08 & 11.49 & -0.41 \\
\hline Medi: & & & & & 195.2 & 184.8 & & & & & & & 12.37 & 11.98 & \\
\hline EE & & & & & 6.57 & 6.72 & & & & & & & 0.63 & 0.64 & \\
\hline
\end{tabular}

* y ** = Significativo a 0.05 y 0.01 de probabilidad; A x B $=$ Cruza directa; B x A = Cruza recíproca; Dif $=$ Diferencia entre los dos tipos de cruzas, EE $=$ Error estándar.

La amplia variabilidad en altura que presentaron las líneas del Grupo QPM se debió principalmente al comportamiento de la línea 12 , que presenta genes de enanismo, ya que en cruzas con líneas enanas originó híbridos de porte bajo; por tal motivo la ACG resultó negativa y demasiado grande (-39.243** y $-49.443 * *$, en cruzas A x B y B x A), en comparación con las demás líneas QPM; es- to influyó significativamente en el rendimiento, debido a la pobre expresión de los híbridos enanos que compitieron con híbridos normales. El comportamiento de la línea 12 fue consistente también en rendimiento de mazorca (REND), al dar valores de -2.455 y -3.985 para las cruzas A x B y B x A, respectivamente (Cuadro 3). 
Cuadro 3. Efectos de aptitud combinatoria general (ACG) de 10 líneas del grupo enano y 9 del grupo QPM (como hembra y macho) para dos variables agronómicas evaluadas en el ciclo agrícola primavera-verano de 2004.

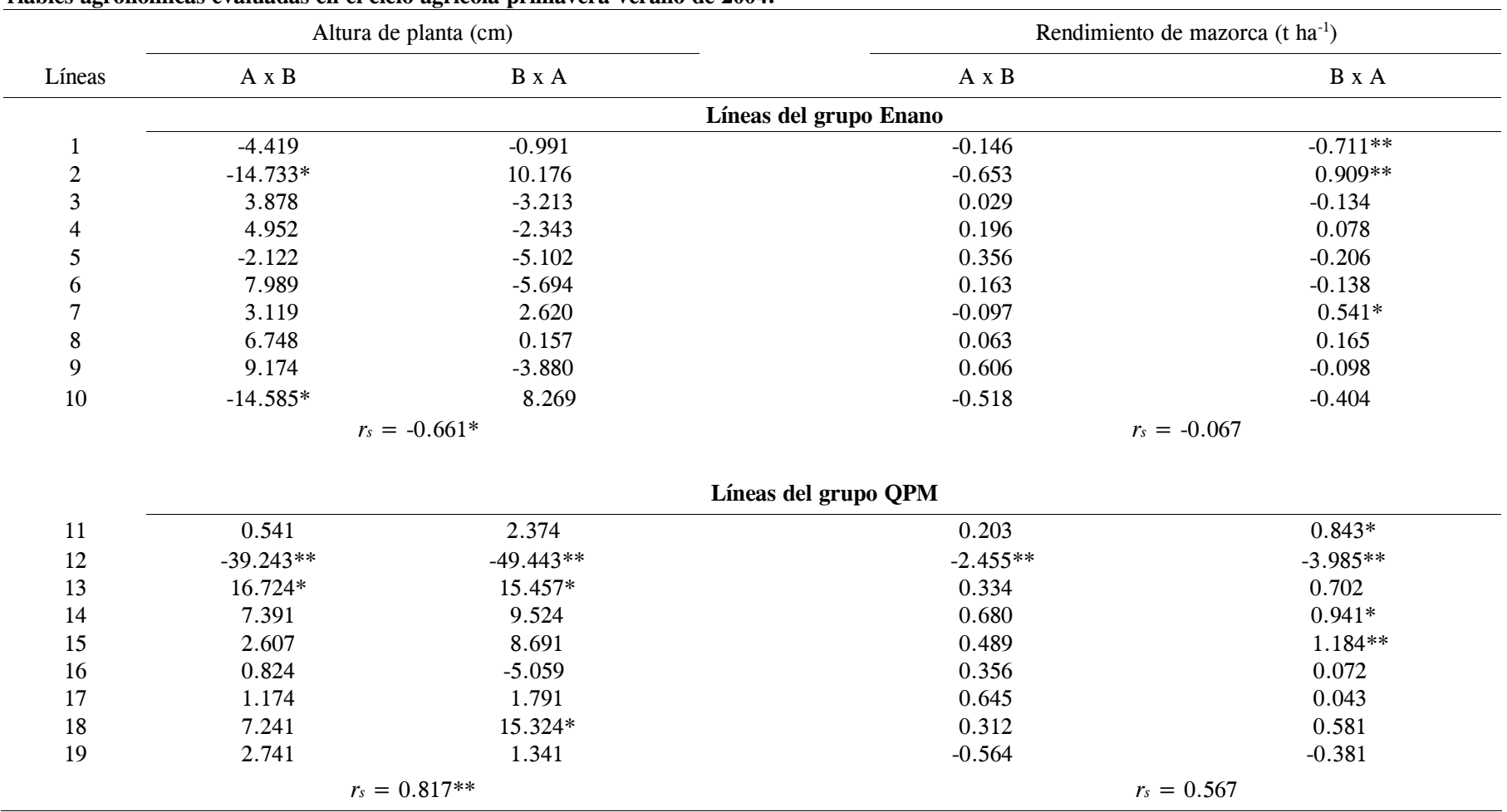

$* \mathrm{y} * *=$ Significativo a 0.05 y 0.01 niveles de probabilidad; $r_{s}=$ Coeficiente de correlación de Spearman.

Los cambios observados en los valores estimados de ACG de las líneas de los Grupos Enano (A) y QPM (B) al invertir el orden de participación en la formación de las cruzas (Cuadro 3), así como en el comportamiento promedio de estos cruzamientos para cada variable de estudio (Cuadro 2), pueden estar influenciados por las aportaciones de los dos grupos de líneas en la expresión de los efectos no-aditivos (Cuadro 1), donde las cruzas A x B obtuvieron el doble de la variación explicada comparado con las cruzas B x A. Los valores estimados de la aptitud combinatoria específica (ACE) de las cruzas A x B y B x A (Figuras 1 y 2 ) para las dos variables de estudio, fueron los más influenciados al invertir la cruza de los progenitores, debido a que las correlaciones fueron del orden de $r_{s}=0.184$ y $r_{s}=0.252 *$ para altura y rendimiento; en esta última, la correlación fue significativa $(\mathrm{P} \leq 0.05)$.

Según Fan et al. (2004), Mickelson et al. (2001) y Vasal et al. (1999), a través de la ACE se pueden clasificar las líneas en grupos heteróticos, con la finalidad de aumentar la heterosis de los híbridos. Sin embargo, con los resultados aquí obtenidos, la clasificación de las líneas no debe realizarse sin tomar en cuenta el orden de cruza- miento que ocupará cada progenitor, ya que de no hacerse así habría sesgo en la selección y, no se obtendrán los resultados deseados.

\section{CONCLUSIONES}

La expresión de las cruzas entre líneas de los dos grupos germoplásmicos estuvo determinada por efectos aditivos de las líneas QPM principalmente, con 70.2 y $61.5 \%$ de la variación en altura de planta y rendimiento de mazorca, respectivamente. Al combinar líneas entre estos dos grupos germoplásmicos no emparentados genéticamente, cambió el orden de participación de los progenitores y determinó diferencias en la expresión fenotípica, y en la estimación de los efectos genéticos de las líneas y sus cruzas. El orden de cruzamiento modificó los valores estimados de aptitud combinatoria general en el grupo Enano y los efectos no-aditivos de los dos grupos de líneas, ya que en la aptitud combinatoria específica se obtuvieron correlaciones del orden de $r_{s}=0.184$ y $r_{s}=0.252 *$ para altura de planta y rendimiento de mazorca. 


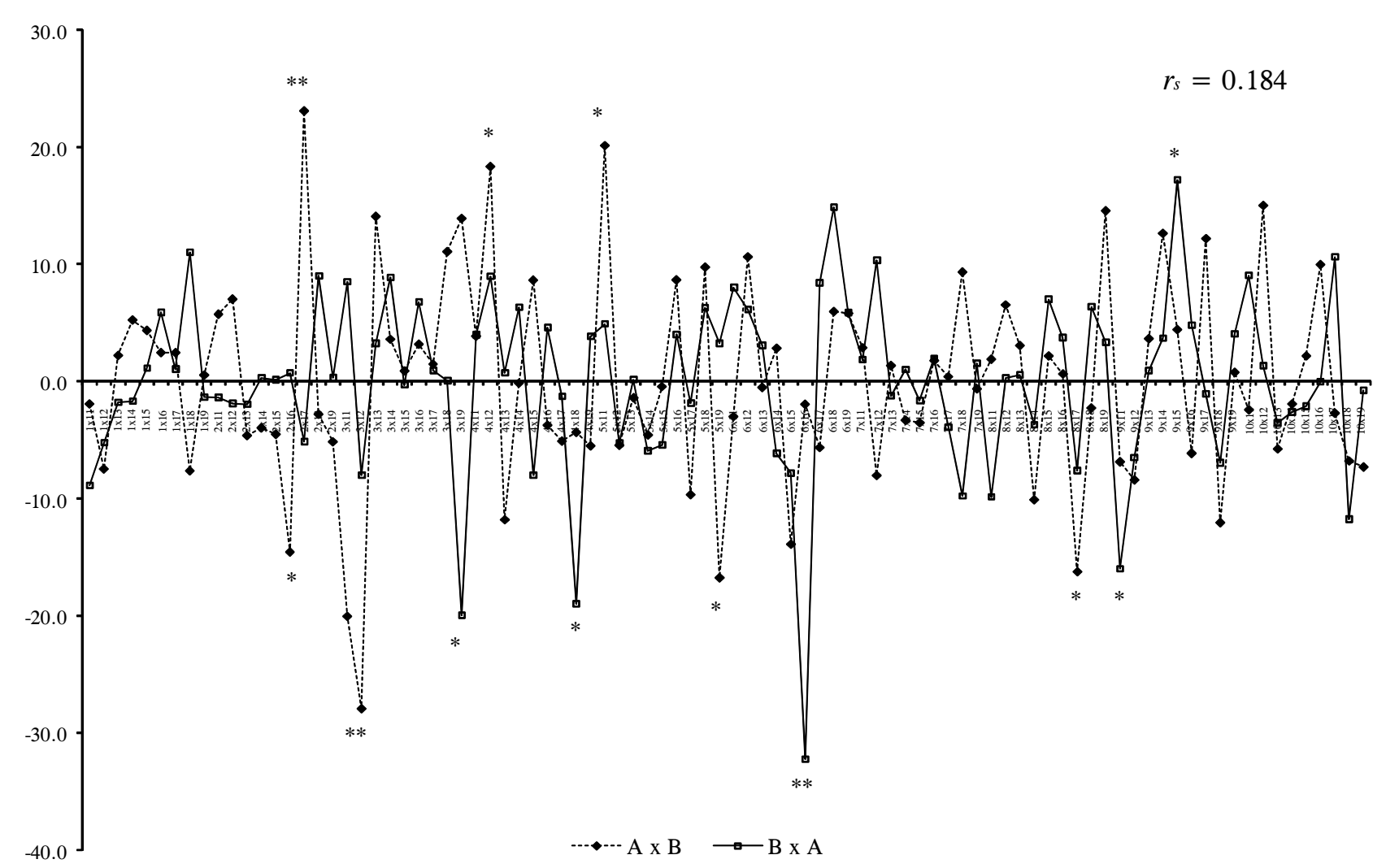

Figura 1. Efectos de aptitud combinatoria específica de 90 cruzas directas $(\mathrm{A} \times \mathrm{x}$ B) y recíprocas $(\mathrm{B} \times \mathrm{X}$ ), formadas por el apareamiento de 10 líneas del Grupo Enano con 9 del Grupo QPM, para altura de planta, evaluadas en el ciclo agrícola primavera-verano 2004 (*,** $=$ Significativo a 0.05 y 0.01 de probabilidad de error, respectivamente).

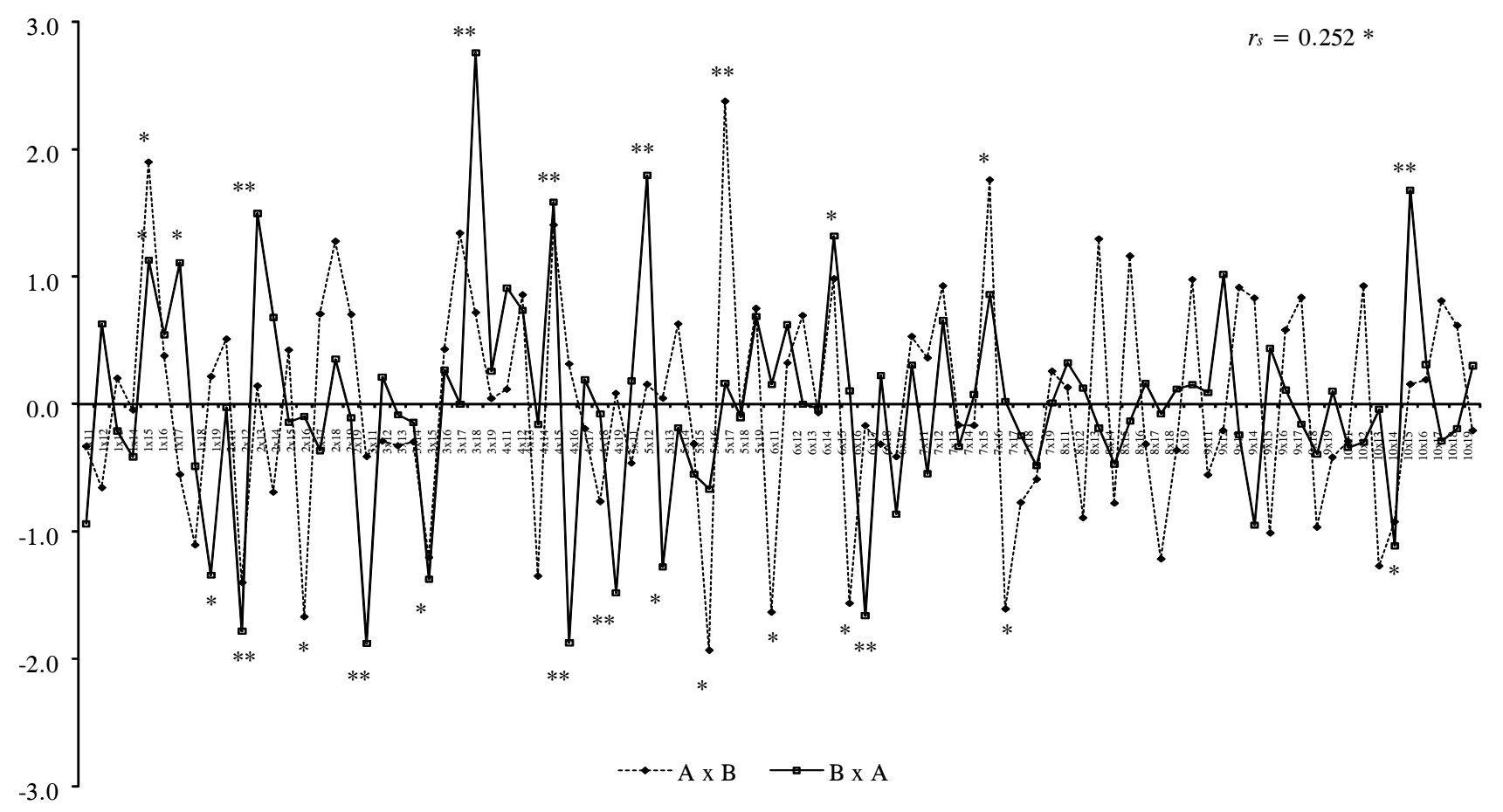

Figura 2. Efectos de aptitud combinatoria específica de 90 cruzas directas $(\mathrm{A} \times \mathrm{B})$ y recíprocas $(\mathrm{B} \times \mathrm{A})$, formadas por el apareamiento de 10 líneas del Grupo Enano con 9 del grupo QPM, para rendimiento de mazorca, evaluadas en el ciclo agrícola primavera-verano $2004(*, * *=$ Significativo a 0.05 y 0.01 de probabilidad de error, respectivamente). 


\section{AGRADECIMIENTOS}

Al Consejo Nacional de Ciencia y Tecnología (CONACYT) por el financiamiento otorgado al proyecto de investigación 41264 correspondiente al presente estudio.

\section{BIBLIOGRAFÍA}

Bernardo R (2001) Breeding potential of intra and interheterotic group crosses in maize. Crop Sci. 41:68-71.

CIMMYT (1998) A complete listing of improved maize germplasm from CIMMYT. Maize Program Special Report. Mexico, D.F. $94 \mathrm{p}$

Corey L A, D F Matzinger, C C Cockerham (1976) Maternal and reciprocal effects on seedling characters in Arabidopsis thaliana (L.) Heynh. Genetics 82:677-683.

Coutiño E B, V A Vidal M (2003) Grain yield stability of corn hybrids using best linear unbiased predictors. Agrociencia 37:605616.

De León C H, F Rincón S, H Reyes V, D Sámano G, G Martínez Z, R Cavazos C, J D Figueroa C (2005) Potencial de rendimiento y estabilidad de patrones heteróticos de maíz formados entre cinco grupos germoplásmicos. Rev. Fitotec. Mex. 28:135-143

Dhliwayo T, K V Pixley, V Kazembe (2005) Combining ability for resistance to maize weevil among 14 Southern African maize inbred lines. Crop Sci. 45:662-667.

Fan X M, J Tan, H M Chen, J Y Yang (2003) Heterotic grouping for tropical and temperate maize inbreds by analyzing combining ability and SSR markers. Maydica 48:251-257.

Fan X M, J Tan, J Y Yang, H M Chen (2004) Combining ability and heterotic grouping of ten temperate, subtropical and tropical quality protein maize inbreds. Maydica 49:267-272.
Haro R P A, M del C Julia, M H Reyes (2007) Determinación materna del contenido de aceite en semillas de girasol. Rev. Fitotec. Mex. 30:39-42.

Hoxha S, M R Shariflou, P Sharp (2004) Evaluation of genetic diversity in Albanian maize using SRR markers. Maydica 49:97 103.

Kang M S, Y Zhang, R Magari (1995) Combining ability for maize weevil preference of maize grain. Crop Sci. 35:1556-1559.

Kang M S, A Kushairi D, Y Zhang, R Magari (1999) Combining ability for rind puncture resistance in maize. Crop Sci. 39:368-371.

Li Y, Y S Shi, YC Song, J Y Du, R Tuberosa, T Y Wang (2004) Analysis of genetic diversity in maize inbred lines based on AFLP markers. Maydica 49:89-95.

Mickelson R H, H Cordova, K V Pixley, M S Bjarnason (2001) Heterotic relationships among nine temperate and subtropical maize populations. Crop Sci. 41:1012-1020.

Pollak L M, S Torres C, A Sotomayor R (1991) Evaluation of heterotic patterns among Caribbean and tropical $\mathrm{x}$ temperate maize populations. Crop Sci. 31:1480-1483.

SAS Institute (2004) SAS/STAT ${ }^{\circledR} 9.1$ User's Guide. Cary, NC: SAS Institute Inc. USA. $5121 \mathrm{p}$.

SAS Institute (2006) Base $\mathrm{SAS} \circledast$ 9.1.3 Procedures Guide. $2^{\text {nd }}$ ed. Vol. 4. Cary, NC. SAS Institute Inc. USA. 398 p.

Singh R K, B D Chaudhary (1985) Biometrical Methods in Quantitative Genetic Analysis. Rev. Ed. Kalyani Publishers. New Delhi - Ludhiana. $318 \mathrm{p}$.

Soengas P, B Ordás, R A Malvar, P Revilla, A Ordás (2003) Performance of flint maize in crosses with testers from different heterotic groups. Maydica 48:85-91.

Vasal K S, H Cordova, S Pandey, G Srinivasan (1999) Tropical maize and heterosis. In: Genetics and Explotation of Heterosis in Crops. American Society of Agronomy, Inc. and Crop Science Society of America, Inc. pp:363-373. 